\title{
Aspectos pedagógicos y curriculares por considerar en el rediseño de un plan de estudios de posgrado con énfasis en docencia universitaria
}

\author{
Curricular and Pedagogical Aspects to Be Taken into Account for Curriculum Redesign \\ and Development in a Master's Program in Higher Education
}

\author{
Aspectos pedagógicos e curriculares para serem tomados em consideração ao \\ redesenhar um currículo de pós graduação com ênfase em docência do ensino superior
}

\author{
Sindy Susana Alvarado-Herrera ${ }^{1}$ \\ Universidad Nacional \\ Heredia, Costa Rica \\ ahcindysusana@gmail.com \\ German Eduardo González-Sandoval² \\ Universidad Nacional \\ Heredia, Costa Rica \\ germangs24@gmail.com \\ Yarina Paniagua-Cortés ${ }^{3}$ \\ Pan-American School \\ Heredia, Costa Rica \\ yarinapaniagua@gmail.com
}

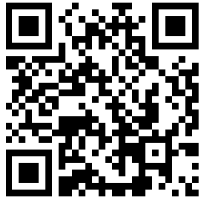

\begin{abstract}
Recibido • Received • Recebido: 07 / 11 / 2016
Corregido • Revised • Revisado: 12 / 02 / 2018

Aceptado • Accepted • Aprovado: 01/ 03 / 2018
\end{abstract}

\begin{abstract}
Resumen: Este trabajo ofrece un abordaje de las diversas opiniones de un grupo de especialistas respecto a la educación superior, con el fin de resaltar los aspectos de importancia que debe considerar la Maestría en Educación con énfasis en Docencia Universitaria de la Universidad Nacional de Costa Rica en el año 2016, como parte de su fortalecimiento y estrategias de mejora en la formación docente, en el marco de las tendencias educativas de internacionalización, movilidad, acreditación, gestión académica y liderazgo compartido. Se parte de un acercamiento a la realidad costarricense en relación con la oferta académica de las maestrías en docencia universitaria, con el propósito de ofrecer una propuesta que responda a las necesidades educativas para la formación en el área de educación superior. Esta es una revisión bibliográfica cuyas fuentes tienen menos de diez años desde su fecha de publicación
\end{abstract}

\footnotetext{
${ }^{1}$ Licenciada en Secretariado Profesional con Énfasis en Educación Comercial y Magíster en Educación con Énfasis en Docencia Universitaria de la Universidad Nacional de Costa Rica. Desde el 2011 se ha desempeñado como académica en la Escuela de Secretariado Profesional de la Universidad Nacional.

${ }^{2}$ Licenciado en Orientación Educativa y Administración Educativa de la Universidad Nacional de Costa Rica y Magíster en Educación con Énfasis en Docencia Universitaria de la Universidad Nacional de Costa Rica. Desde 2007 se ha desempeñado como docente, investigador, extensionista y actualmente como director de la División de Educación para el Trabajo del Centro de Investigación y Docencia en Educación de la Universidad Nacional.

${ }^{3}$ Magíster en Educación con énfasis en Aprendizaje del Inglés y Magíster en Educación con énfasis en Docencia Universitaria de la Universidad Nacional de Costa Rica. Se desempeña como docente de inglés: lengua y literatura en Pan-American School.
} 
doi: http://dx.doi.org/10.15359/ree.22-2.9

URL: http://www.una.ac.cr/educare

CORREO: educare@una.cr

y son referentes importantes de las tendencias educativas que guían esta producción académica. Además, como parte de la contextualización, se consideran los programas de maestría en docencia universitaria que ofrecen las universidades en Costa Rica. Producto del análisis de estos aportes se concluye la pertinencia de los programas de docencia en educación superior, particularmente el de la Universidad Nacional, así como la necesidad de fortalecer su divulgación mediante procesos integrales que atiendan los criterios de calidad para programas de posgrado mencionados anteriormente.

Palabras claves: Acreditación; cooperación universitaria; enseñanza superior; formación de docentes; grado de maestría, investigación; movilidad.

Abstract: This project addresses the publications of a group of renowned authors in order to highlight important aspects to be considered by the Education Master's Program in Higher Education and Teaching of the National University of Costa Rica as part of its improvement strategies in teacher education, taking into account the most recent tendencies related to internationalization, mobility, accreditation, academic management and shared leadership in higher education. The paper aims to portray a proposal that fulfills the needs of the Costa Rican master's programs in higher education and teaching by approaching this country's reality in terms of what the programs offer. It is a bibliographic research paper, which sources have been published for no longer than 10 years and are truly groundbreaking in regard to the graduate program trends that guide this production. Moreover, as part of the contextualization needed, all master's degrees in higher education and teaching offered in the country are taken into consideration. As a result of this research, it can be concluded that these programs are pertinent, especially the one offered by the National University of Costa Rica, and it is evident that disseminating the program's highlights and achievements through processes that respond to quality criteria for graduate programs truly needs to be encouraged.

Keywords: Accreditation; higher education; master's degrees; mobility; research; teacher education; university cooperation.

Resumo: Este artigo apresenta uma abordagem das diferentes opiniões de um grupo de especialistas sobre o ensino superior, a fim de destacar os aspectos importantes a considerar no Mestrado em Educação com ênfase em docência, do ensino superior da Universidade Nacional da Costa Rica, no ano de 2016. Estes aspectos fazem parte das estratégias e fortalecimento em aperfeiçoar a formação de professores, como parte das tendências educacionais de internacionalização, mobilidade, acreditação, gestão académica e liderança compartilhada. Como tema inicial, se faz uma aproximação à realidade da Costa Rica em relação às ofertas acadêmicas de graus de mestrado em docência do ensino superior, com o objetivo de oferecer uma proposta que atenda às necessidades educacionais de formação na área do ensino superior. Parte desta é uma revisão de literatura, cujas fontes são menores a dez anos, a partir da data de sua publicação, e são referências importantes de tendências educacionais que orientam essa produção acadêmica. Além disso, como parte da contextualização, são considerados os programas de mestrado em docência do ensino superior oferecidos por universidades na Costa Rica. Como resultado das análises destas contribuições, a conclusão demostra a relevância dos programas de docência do ensino superior, particularmente os oferecidos pela Universidade Nacional, bem como a necessidade de reforçar a sua divulgação através de processos integrados que atendem aos critérios de qualidade para os programas de pós-graduação acima mencionados.

Palavras-chave: Cooperação universitária; acreditação; ensino superior; formação de professores; mestrado; mobilidade. 


\section{Introducción}

La formación de los grupos docentes universitarios es una necesidad evidente para afrontar el desarrollo de la educación superior. En el contexto costarricense, esta labor es asumida únicamente por tres programas de maestría ofrecidos por la Universidad Nacional, la Universidad de Costa Rica y la Universidad Latinoamericana de Ciencia y Tecnología. En búsqueda de aportar a la actualización e innovación como proceso de mejora del programa de posgrado de la Maestría en Educación con Énfasis en Docencia Universitaria, la cual pertenece a la División de Educología de la Universidad Nacional de Costa Rica, así como a otros programas similares, se realiza una revisión bibliográfica acerca de aspectos pedagógicos y curriculares en los cuales diversos estudios Ilaman la atención como líneas de acción que deben ser parte de esos cambios a los cuales están llamadas las universidades que imparten programas de posgrado.

Algunos de estos textos se enmarcan, además, en los acuerdos plasmados en la "Declaración de Bolonia" (1999), como resultado de una amplia discusión y basada en la atención de las necesidades de mejora educativa en los países europeos, declaración conjunta por parte de los Ministerios Europeos de Educación.

También se realiza un acercamiento a la realidad nacional en términos de internacionalización, plasmada en el Quinto Informe del Estado de la Educación (Programa Estado de la Nación, 2015).

Al ser el Centro de Investigación y Docencia en Educación (CIDE), la División de Educología y, en consecuencia, la Maestría en Educación, referentes en el ámbito educativo costarricense, se hace necesario realizar esta revisión de aspectos importantes e innovadores (internacionalización, movilidad, acreditación, gestión académica y liderazgo compartido) con los cuales debe cumplir un programa de posgrado en el marco de los cambios educativos del siglo XXI. Para ello, se inicia con la revisión del planteamiento de los ejes curriculares sugeridos para los programas de posgrado y, específicamente, del objetivo del énfasis de docencia universitaria de la Maestría en Educación, que se describe en la página web de la Universidad Nacional (s. f., párr. 1) como: "Ofrecer formación profesional para el desempeño en instituciones de educación superior, en lo relativo al área académica de la docencia universitaria, que es abordada desde una visión integral que reconoce su complejidad y la necesidad de su abordaje interdisciplinario".

Por lo tanto y en atención a la responsabilidad institucional de ofrecer formación profesional para el desempeño de la docencia universitaria, se considera oportuna esta revisión bibliográfica para favorecer la reflexión de la temática en la Universidad Nacional de Costa Rica y también como insumo para otras instituciones u organizaciones que ofrecen y desarrollan posgrados de docencia universitaria. 
doi: http://dx.doi.org/10.15359/ree.22-2.9

URL: http://www.una.ac.cr/educare

CORREO: educare@una.cr

\section{Metodología}

Esta indagación implicó la consulta de información institucional, libros en línea y artículos publicados en revistas académicas indexadas en el buscador Google Académico, tales como la Revista Gestión de la Educación, Revista de Universidad y Sociedad del Conocimiento, Revista Educere et Educare, Núcleo de estudios e investigaciones en educación superior de MECOSUR, Debate Universitario, Educación, Arbor CLXXIII, Revista Ciencia y Tecnología, Revista Iberoamericana sobre Calidad, Eficacia y Cambio en Educación, Revista Iberoamericana de Evaluación Educativa, entre otras. Estos artículos tienen, en su mayoría, menos de 10 años de haber sido publicados, por lo que se considera son lo suficientemente recientes para los efectos de esta investigación. La información consultada fue organizada en diferentes temáticas relacionadas con la actualidad y el futuro de los posgrados en educación con énfasis en docencia universitaria, a saber: ejes curriculares, formación y prácticas docentes, internacionalización y movilidad, gestión académica y liderazgo compartido, y acreditación.

\section{Ejes curriculares sugeridos para los programas de posgrado}

Los programas de posgrado en docencia universitaria deben trabajar en la formación de profesionales capaces de proponer, generar y compartir conocimiento, a partir del cual logre transformar las realidades de aula y, como consecuencia, la realidad social y cultural de su comunidad, contribuyendo a la construcción de una mejor sociedad. Por tanto, el currículo es uno de los aspectos por revisar y respecto al cual se repasa la propuesta de estructuración curricular de una maestría, planteada por Lucarelli (2004) basada en los siguientes tres ejes:

- Eje disciplinar: está estructurado en Unidades referidas a: La Universidad: "grupos, organizaciones e instituciones"; Contexto social y Universidad; Currículum Universitario: demandas y propuestas; El Currículum en acción: la enseñanza universitaria y La gestión en el aula en su relación con otras funciones de investigación, extensión y transferencia.

- Ejedeindagación sobrelas prácticas:contiene Unidades relativasa Laformación deequipos en el gobierno universitario; Las modalidades de organización académica y La gestión de enseñanza en el aula. Permite a las personas participantes desarrollar conocimientos y habilidades que posibiliten abordar reflexivamente situaciones de su práctica como docente universitario en los distintos ámbitos institucionales, por lo que estas Unidades asumen la modalidad de observaciones no participantes, prácticas en terreno, pasantías, laboratorios de análisis de la propia práctica, con instancias de recuperación crítica.

- Eje de investigación: está integrado por dos Cursos de Metodología de la Investigación, y un tercer Espacio Curricular para trabajar los problemas de la Articulación entre investigación, enseñanza y extensión, en el que se presentan oportunidades de tareas de investigación, con tutorías. (p. 518) 
El plan de estudios de la Maestría en Educación con énfasis en Docencia Universitaria contempla tres áreas entre las cuales se distribuyen las materias que imparte, a saber: tronco común (materias pedagógicas), cursos del énfasis (docencia universitaria) e investigación. Cada una de estas áreas responde a los ejes curriculares planteados por Lucarelli (2004). Sin embargo, el eje de indagación sobre prácticas puede reforzarse desde la perspectiva de este autor.

A partir de lo anterior, se puede decir que la estructura curricular de esta contempla la integración de los anteriores ejes curriculares, logrando con ello una propuesta de calidad y holística para la formación de formadores, por cuanto considera la disciplina propiamente, las prácticas docentes y la investigación. Al respecto, Abreu, 2009 (citado por Dávila, 2012) argumenta:

Una formación de calidad requiere múltiples interrelaciones entre el mundo académico y el profesional. Para esto es necesario superar concepciones que plantean límites rígidos entre la docencia, la investigación y la práctica profesional. La transformación en la enseñanza de posgrado debe implicar un equilibrio entre investigación y formación. (p. 21)

Considerando que este programa de Maestría mantiene un balance entre los puntos citados previamente, se detallan a continuación los aspectos más relevantes que debe fortalecer la Maestría en Educación para atender las necesidades educativas del siglo XXI, desde la perspectiva crítica de quienes escriben.

\section{Formación y prácticas docentes}

Uno de los problemas que plantea el sistema educativo universitario es la falta de formación en el área pedagógica o andragógica de muchas personas que ejercen la labor docente, personas reconocidas por poseer amplios conocimientos y experiencia en su área disciplinar de formación. Al respecto, Lucarelli (2004) manifiesta:

En este entorno el docente universitario es reconocido por el alto nivel de preparación académica y profesional en su campo disciplinar, sin que esta formación necesariamente esté acompañada por conocimientos específicos sobre la práctica de la enseñanza, que le permitan dar una respuesta adecuada y científicamente fundamentada, a las nuevas problemáticas que afectan al aula universitaria. (pp. 505-506)

Siendo la formación pedagógica y andragógica una de las principales funciones que realiza el CIDE, la Maestría en Educación con énfasis en Docencia Universitaria nace como una propuesta para solventar la necesidad de formación de docentes de la Universidad Nacional. Con ello se busca reducir el número de personal académico que carece del conocimiento en estas disciplinas y contar con personal docente que desarrolle las competencias necesarias para el desempeño de su profesión con calidad, tal como lo plantean Salomón y Amador (2013): 
doi: http://dx.doi.org/10.15359/ree.22-2.9

URL: http://www.una.ac.cr/educare

CORREO: educare@una.cr

Las universidades deben aspirar a capacitar pedagógicamente a los docentes que integran la estructura de diseño y desarrollo curricular, recordando que ellos son especialistas en sus áreas, no en currículo, y, por lo tanto, se deben proporcionar los elementos técnicos que ellos deberán aplicar en sus respectivas áreas del conocimiento. (p. 10)

Se considera que, para atender esta propuesta de capacitación pedagógica para el personal docente universitario, es importante que la Universidad Nacional genere conciencia en sus profesionales para que continúen formándose en docencia universitaria y que existan mecanismos permanentes de divulgación para dar a conocer la oferta académica de esta maestría, tanto a nivel nacional como internacional.

Así mismo, es necesario reforzar los procesos de actualización profesional mediante la capacitación y formación continua a nivel institucional para que permita favorecer las buenas prácticas docentes y la divulgación de estas mismas.

\section{Investigación y extensión}

Una de las mayores falencias de la educación superior es la falta de formación para realizar investigación desde sus primeras etapas, una labor que se empieza a profundizar en los programas de posgrado, lo cual dificulta la ampliación de esta actividad a otros ámbitos en los cuales es necesaria. Así lo afirman Salomón y Amador (2013):"un desafío adicional se relaciona con la creciente necesidad de hacer investigación interdisciplinaria, interuniversitaria e internacional" (p. 6).

Si se da un fortalecimiento de la investigación en las primeras etapas de la educación superior, incluso en la educación secundaria, se favorecería la práctica investigativa en las formas propuestas, sea interdisciplinaria, interuniversitaria e internacional, incluso entre grupos de docentes y estudiantes, como una forma dinámica de hacer la función académica.

Es importante reconocer que la Maestría en Educación fomenta la investigación y producción de nuevo conocimiento, tanto en el estudiantado como en el profesorado, con lo cual se evidencia un liderazgo en la acción sustantiva institucional. formación académica, producción y divulgación. Sin embargo, se considera necesario fortalecer la actividad investigativa como eje transversal en los programas de maestría, así como la escritura académica, que permita a sus estudiantes mejorar los conocimientos y destrezas de investigación para escribir sus artículos académicos. Al respecto Tesouro, Corominas, Teixidó y Puiggalí (2014) afirman:

Toda formación pedagógica puede mejorar la autoeficacia docente y repercutir en los enfoques de enseñar. Puesto que consideramos que fortalecer el vínculo docenciainvestigación constituye un elemento importante en la acción de autoperfeccionamiento de todo profesor universitario, entendemos que hace falta actuar de manera integrada en la acción profesional-personal del profesorado. El marco idóneo para un primer nivel de sensibilización, convencimiento y práctica es el propio departamento. (p. 183) 
Por otra parte, el programa de Maestría debe promover la extensión que, como producto de la investigación, pueda realizar aportes significativos a la comunidad nacional, con los cuales logre articular las acciones necesarias con el Estado y otros sectores sociales que le permitan ser objeto de apoyos, convenios y alianzas para impulsar especialidades o investigaciones en temas que coincidan con la agenda nacional y la agenda universitaria (Salomón y Amador, 2013). Esta vinculación social, producto de la investigación, debe ser un compromiso asumido por estudiantes, docentes y personal administrativo de la Maestría en Educación.

\section{Acreditación}

La acreditación de carreras es una de las tendencias más destacadas en educación superior en los últimos tiempos; este es un requerimiento del estudiantado para la elección de sus carreras por tratarse de una garantía de calidad y excelencia que, además, favorece su formación y es un factor determinante para su empleabilidad. González y Santamaría (2013) definen la acreditación en los siguientes términos:

La evaluación que da fe pública de la calidad de un programa, una dependencia o una institución, con base en determinados estándares genéricos previamente establecidos por un organismo externo, sea nacional, regional, internacional o de otro tipo; el grado de cumplimiento de dichos estándares da lugar a una calificación usualmente expresada en términos temporales (un determinado número de años) y puede o no estar acompañada de observaciones o aspectos a corregir. (p.139)

Tomando en cuenta lo anterior, uno de los aspectos que la Maestría en Educación de la Universidad Nacional debe considerar es su acreditación con alguna de las diferentes agencias que existen para ello. Esto, porque de acuerdo con la Agencia Centroamericana de Acreditación de Posgrado (ACAP, por sus siglas en español), mediante este proceso se evaluarán de manera sistemática criterios como: mejoramiento continuo, pertinencia, impacto, coherencia, eficiencia, equidad, idoneidad, rigurosidad científica, transparencia e independencia. Para ello, ACAP establece ocho categorías que deben evaluar los posgrados, a saber: estudiantado, estudiantado graduado, profesorado, procesos formativos, investigación e innovación, gestión académica y administrativa, infraestructura y recursos de apoyo al programa; vinculación, proyección e incidencia social; y colaboración e intercambio académico a nivel nacional, regional e internacional (Agencia Centroamericana de Acreditación ACAP, 2008).

La acreditación conlleva un proceso a través del cual se realiza la revisión detallada y profunda de los programas de estudio y demás aspectos relacionados con la calidad del servicio que ofrece un programa educativo, de forma que alcance estándares internacionales. González y Santamaría (2013) mencionan los siguientes objetivos compartidos por diferentes organismos 
doi: http://dx.doi.org/10.15359/ree.22-2.9

URL: http://www.una.ac.cr/educare

CORREO: educare@una.cr

como UDUAL (Unión de Universidades de América Latina y el Caribe) y RIEV (Red Internacional de Evaluadores) con respecto a la acreditación internacional:

- Incrementar la credibilidad social de las IES [instituciones de educación superior].

- Mejorar las instituciones o programas.

- Generar estándares internacionales orientados a mejorar la competitividad.

- Facilitar la obtención de recursos económicos.

- Promover el reconocimiento internacional de títulos profesionales mediante sistemas de equivalencia de créditos o cargas horarias.

- Fomentar la movilidad estudiantil y de profesionales entre los diferentes países. (p.141)

Al igual que UDUAL y RIEV, la ACAP tiene los siguientes objetivos, en procura del mejoramiento de la educación superior:

a. Promover el mejoramiento continuo de la calidad y de la pertinencia de los programas de postgrado.

b. Acreditar la calidad de los programas de posgrado tanto [del] ámbito nacional como regional.

c. Obtener y mantener la acreditación y el reconocimiento, como ACAP, ante el Consejo Centroamericano de Acreditación (CCA) y otros organismos.

d. Consolidar y promover la participación de instituciones y de sectores interesados en la calidad de los estudios de posgrado.

e. Contribuir permanentemente con el desarrollo centroamericano y con la integración regional. (ACAP, 2008, p. 12)

Enfatizando la importancia de que las instituciones de educación superior, y específicamente los programas de posgrado, atiendan estas tendencias desde una posición ética en procura de realizar procesos de mejoramiento permanente y garantizar una educación de calidad, citamos las conclusiones de Contreras, Alvarado, Maldonado y Benítez (2009):

Las instituciones de educación superior son organizaciones que se han visto obligadas a cambiar, conforme la sociedad y organismos reguladores se lo señalan. Contar con programas de posgrado como parte de la oferta educativa es trascendental para las universidades, ya que a través de éstos es posible cumplir con una de sus funciones 
sustantivas (generación y aplicación de nuevos conocimientos a través de la investigación); sin embargo, se requiere que estos programas y su operación se realicen con el pleno conocimiento de sus principales responsables, respecto a lo que es necesario hacer para el aseguramiento de la calidad. (p. 42)

Finalmente, la acreditación representa un reto para los programas de posgrado, pues les genera beneficios como el mejoramiento de sus programas, desarrollo permanente, reconocimiento y credibilidad internacional, obtención de recursos, movilidad entre sus estudiantes y personal académico, entre otros.

\section{Internacionalización y movilidad}

Con los cambios sociales producto de la globalización, atendiendo además las tecnologías que ampliaron las fronteras, se empezaron a realizar esfuerzos por trabajar de forma conjunta entre países para lograr su desarrollo y fortalecimiento. De ahí que en lugares como Europa se hayan originado importantes cambios que dan paso a las propuestas de internacionalización y movilidad, como es el ejemplo del proceso de Bolonia que da lugar a la declaración del mismo nombre ("Declaración de Bolonia", 1999). Dávila (2012) explica que el "principal objetivo del proceso de Bolonia es la búsqueda de áreas de convergencia entre los sistemas europeos de Educación Superior" (p. 19).

[Es ahí donde se establecen acuerdos para la] promoción de la necesaria dimensión europea en la enseñanza superior, especialmente por lo que respecta a la elaboración de programas de estudios, la cooperación interinstitucional, los programas de movilidad y los programas integrados de estudios, formación e investigación. ("Declaración de Bolonia", 1999, p. 3)

Uno de los conceptos clave en que se basan estas propuestas es la globalización, donde se plantea la disminución de brechas entre países, lo cual implica en educación superior la búsqueda de alianzas, como propone Ferro (2014):

La necesidad de entenderse con otros interlocutores del mundo académico internacional nos orienta hacia la búsqueda de alianzas con centros universitarios de mayor fortaleza, con comunidades científicas poseedoras de mayores recursos para realizar proyectos comunes, tanto en el campo de la formación universitaria como en el de la investigación y la cultura, incluyendo los servicios al sector externo. (p. 13)

Con respecto a uno de los seis objetivos generales que plantea la Declaración de Bolonia, Ortega (2002) dice que "el primero señala la necesidad de 'adoptar un sistema de grados 
doi: http://dx.doi.org/10.15359/ree.22-2.9

URL: http://www.una.ac.cr/educare

CORREO: educare@una.cr

fácilmente entendible y comparable para promover la empleabilidad de los ciudadanos europeos y la competitividad del sistema europeo de educación superior"' (p. 72). Al respecto se hace hincapié en la importancia de lo que plantea este objetivo, al facilitar el reconocimiento de los estudios para que las personas puedan movilizarse a otras zonas y obtengan la equiparación de los estudios y preparación ya recibida, de manera que se plasma el tema de la internacionalización como una de las funciones permanentes de las universidades.

En el marco costarricense, Mora (2015, citado por el Programa Estado de la Nación, 2015) menciona que la internacionalización incluye:

a) la convergencia de sistemas de educación terciaria y acuerdos de reconocimiento internacional o armonización de la educación; b) la internacionalización de contenidos de los programas y de la oferta académica; c) la movilidad de profesionales académicos y estudiantes con fines formativos y para promover la interculturalidad, impulsados a través de becas, convenios y otros, y generalmente, la parte del concepto que más se asocia con la internacionalización; y por último, d) la movilidad de instituciones educativas y sus programas (mediante sucursales, educación virtual, programas conjuntos, doble titulación otros). (p. 228)

Además, según el Quinto Informe del Estado de la Educación, dadas las características del sistema de educación superior de Costa Rica, no "existe un marco normativo y político para la promoción de la internacionalización..." (Programa Estado de la Nación, 2015, p. 228). No obstante, se identifican "cuatro formas de internacionalización de la educación superior costarricense: la armonización de los sistemas y los acuerdos para la internacionalización y el reconocimiento mutuo, la internacionalización en la oferta académica y la investigación, la movilidad de las personas y las redes, y la movilidad de instituciones y programas (educación transfronteriza)" (Programa Estado de la Nación, 2015, p. 228).

Al respecto, Aguilar-Castillo y Riveros-Angarita (2015) comentan el caso de la internacionalización en Costa Rica, la cual tomó fuerza en 2005 con el Plan Nacional Superior de la Educación Universitaria Estatal (PLANES 2006-2010), que contempla "la capacidad de las universidades para responder a las demandas nacionales e internacionales" (p. 106). Además, las autoras indican:

En el caso de Costa Rica, el área de internacionalización y cooperación académica internacional en cada una de las cinco universidades estatales posee su respectiva oficina ... de relaciones internacionales: Universidad de Costa Rica (Oficina de Asuntos Internacionales y Cooperación Externa), Universidad Nacional (Oficina de Cooperación Técnica Internacional), Instituto Tecnológico de Costa Rica (Dirección de Cooperación), 
Universidad Estatal a Distancia (Dirección de Internacionalización y Cooperación) y Universidad Técnica Nacional (Dirección de Cooperación Externa). De igual manera, algunas de las 52 universidades privadas que se encuentran funcionando en Costa Rica cuentan con sus oficinas de relaciones internacionales con diferente grado de desarrollo. (p. 107)

En el marco de la internacionalización y movilidad, el programa de Maestría en Educación del CIDE se ha preocupado por promover la participación de personal académico destacado que viene de universidades del extranjero, con lo cual se cumple con una parte de la propuesta de movilidad, que brinda oportunidades de estudio y formación en otros contextos culturales, tanto para estudiantes, profesorado y equipos de investigación, y permite el fortalecimiento de las actividades académicas.

No obstante, en el contexto latinoamericano, estos procesos se han visto dificultados, según lo explican Del Bello y Mundet (2004, citados por Dávila, 2012):

A. Limitada o nula compatibilidad de los sistemas nacionales;

B. Inflexibilidad curricular, escasa interdisciplinariedad, proliferación de títulos y extensa duración de las carreras de grado;

C. Marcos regulatorios restrictivos que acotan la autonomía universitaria, dado que las regulaciones estatales intervienen en la validez nacional de los títulos e incluso, en muchos casos, en los contenidos curriculares;

D. Limitados sistemas nacionales de aseguramiento de la calidad, aunque en los últimos años se han creado agencias nacionales de evaluación de la calidad universitaria;

E. Reserva de mercado para profesionales universitarios nacionales. (p. 24)

Estas diferencias se pueden observar incluso en entornos nacionales, donde algunas universidades ofrecen la misma carrera con diferentes enfoques, duración y otras características, tal como se evidencia en la revisión del programa de Maestría en Educación con énfasis en Docencia Universitaria impartido por la Universidad Nacional, así como sus similares de la Universidad de Costa Rica y la Universidad Latinoamericana de Ciencia y Tecnología, todas en el contexto costarricense. Estos programas de maestría presentan discrepancias importantes respecto a la propuesta de maestría académica o profesional, el plan de estudios, la duración del posgrado y el perfil de salida del estudiantado, según se puede apreciar la Tabla 1: 
doi: http://dx.doi.org/10.15359/ree.22-2.9

URL: http://www.una.ac.cr/educare

CORREO: educare@una.cr

Tabla 1: Comparación de programas de Maestrías en Docencia, universidades costarricenses

\begin{tabular}{|c|c|c|c|}
\hline Característica & Universidad Nacional (UNA) & Universidad de Costa Rica (UCR) & $\begin{array}{c}\text { Universidad Latinoamericana de Ciencia } \\
\text { y Tecnología (ULACIT) }\end{array}$ \\
\hline Tipo de universidad & Pública & Pública & Privada \\
\hline Nombre del posgrado & $\begin{array}{l}\text { Maestría en Educación, con énfasis } \\
\text { en Docencia Universitaria }\end{array}$ & $\begin{array}{l}\text { Maestría Académica en } \\
\text { Educación, con énfasis en } \\
\text { Docencia Universitaria }\end{array}$ & $\begin{array}{l}\text { Maestría en Currículum y Docencia } \\
\text { Universitaria }\end{array}$ \\
\hline Tipo de posgrado & Profesional & Académico & No indica \\
\hline Duración de la carrera & $\begin{array}{l}6 \text { trimestres y } 1 \text { curso de verano } \\
2 \text { años }\end{array}$ & $\begin{array}{l}5 \text { ciclos semestrales y } 2 \text { cursos de } \\
\text { verano } \\
2,5 \text { años (más tesis) }\end{array}$ & $\begin{array}{l}3 \text { cuatrimestres } \\
1 \text { año }\end{array}$ \\
\hline Cantidad de cursos & 19 cursos & 18 cursos & 16 cursos \\
\hline Perfil & $\begin{array}{l}\text { Perfil del estudiantado graduado } \\
\text { Obtendrá conocimiento sobre: } \\
\text { a. Los antecedentes históricos } \\
\text { de la educación superior y la } \\
\text { evolución de su estructura y } \\
\text { funcionamiento. } \\
\text { b. Las teorías y principios } \\
\text { fundamentales que sustentan la } \\
\text { educación de personas adultas } \\
\text { (andragogía) y su relación con la } \\
\text { educación superior. } \\
\text { c. Los procesos y factores que } \\
\text { inciden en el aprendizaje a nivel } \\
\text { de la educación superior. } \\
\text { d. Los procesos y estrategias de } \\
\text { enseñanza y aprendizaje y su } \\
\text { relación con la planificación } \\
\text { curricular. } \\
\text { Adquirirá habilidades y } \\
\text { destrezas para: } \\
\text { a. Aplicar conceptos, principios } \\
\text { y técnicas en la formulación } \\
\text { y evaluación de experiencias } \\
\text { innovadoras en la educación } \\
\text { superior. } \\
\text { b. Aplicar estrategias didácticas en } \\
\text { la educación superior. } \\
\text { c. Utilizar instrumentos de } \\
\text { evaluación de conformidad con } \\
\text { los objetivos y actividades de } \\
\text { aprendizaje. } \\
\text { d. Organizar la docencia de } \\
\text { conformidad con los estilos de } \\
\text { aprendizaje del estudiantado. }\end{array}$ & $\begin{array}{l}\text { Perfil profesional } \\
\text { Formamos profesionales en } \\
\text { respuesta a los requerimientos } \\
\text { actuales de la investigación de } \\
\text { la docencia universitaria, con la } \\
\text { intencionalidad de reflexionar y } \\
\text { profundizar en desarrollar tanto } \\
\text { la plataforma conceptual como } \\
\text { la investigativa y contribuir } \\
\text { con el mejoramiento de la } \\
\text { actividad académica del docente } \\
\text { universitario. (Universidad de } \\
\text { Costa Rica,SEP, s. f., párr. 1) }\end{array}$ & 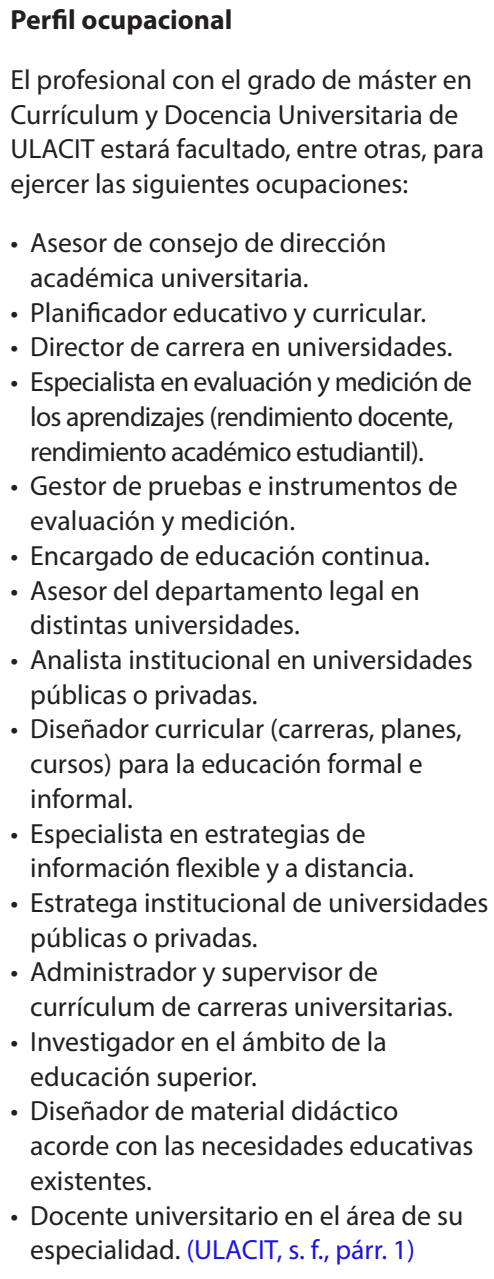 \\
\hline
\end{tabular}

continúa 


\begin{tabular}{|c|c|c|c|}
\hline Característica & Universidad Nacional (UNA) & Universidad de Costa Rica (UCR) & $\begin{array}{c}\text { Universidad Latinoamericana de Ciencia } \\
\text { y Tecnología (ULACIT) }\end{array}$ \\
\hline & $\begin{array}{l}\text { Poseerá las siguientes actitudes: } \\
\text { a. Creatividad, en el desarrollo } \\
\text { del quehacer docente, como } \\
\text { elemento fundamental para el } \\
\text { mejoramiento de la docencia } \\
\text { universitaria. } \\
\text { b. Interés constante, por } \\
\text { la actualización y el } \\
\text { perfeccionamiento docente. } \\
\text { c. Flexibilidad para asumir las } \\
\text { diferencias que se presentan } \\
\text { en distintas situaciones de } \\
\text { enseñanza y aprendizaje. } \\
\text { d. Capacidad para valora y } \\
\text { compartir las experiencias } \\
\text { innovadoras en los programas } \\
\text { y modalidades de docencia } \\
\text { universitaria. (S. Vega, } \\
\text { comunicación personal, } 6 \text { de } \\
\text { febrero, 2018). }\end{array}$ & & \\
\hline
\end{tabular}

Nota: Tabla elaborada con base en información publicada por las universidades en sus sitios web, acerca de sus programas de maestría en docencia universitaria (Universidad de Costa Rica, SEP, s. f.; ULACIT, s. f.), así como una comunicación personal sobre el plan de estudios de la Maestría en Educación con énfasis en Docencia Universitaria de la Universidad Nacional (S. Vega, comunicación personal, 6 de febrero, 2018).

Tal como se muestra en la Tabla 1, existen discrepancias desde el tipo de universidad que ofrece el programa en Costa Rica: dos de ellas son públicas mientras una es privada. Aunque existe cierta similitud entre la duración del programa de la UNA y el de la UCR ( 2 años y 2,5 años, respectivamente), se puede observar que la maestría de la ULACIT puede terminarse en la mitad del tiempo (o incluso menos) que sus homólogos. En términos del tipo de posgrado, una de las universidades lo ofrece en la modalidad profesional, otra en la académica y la ULACIT no indica en cuál de estas se ubica su programa de maestría. Por otra parte, se aprecian coincidencias respecto al número de cursos incluidos: 19, 18 y 16 para cada una de las maestrías. Finalmente, en referencia al perfil del estudiantado graduado, la UNA lo presenta en términos de conocimientos y actitudes, la UCR de intencionalidades (reflexionar, contribuir, entre otras) hacia la labor del personal docente universitario y finalmente la ULACIT lo presenta como posibles puestos de trabajo en los que el estudiantado graduado se pueda desempeñar.

De esta forma se puede concebir que, si en un país tan pequeño como Costa Rica, y tomando en cuenta solamente tres instituciones de educación superior, los programas de maestría en docencia universitaria son tan distintos; a nivel internacional estas desigualdades son aún más marcadas, si se consideran las diferencias culturales e incluso de la fundamentación filosófica de cada una de las instituciones. Casas (2005), al respecto, comenta: 
doi: http://dx.doi.org/10.15359/ree.22-2.9

URL: http://www.una.ac.cr/educare

CORREO: educare@una.cr

El área iberoamericana posee un gran numero [sic] de universidades y de instituciones de educación superior, pero todas ellas con niveles de calidad y organizativos sumamente heterogéneos. Además, dentro de la clasificación de educación superior, se ubica una enorme variedad de organizaciones para estudios y especializaciones, que generalmente emiten títulos refrendados por los respectivos gobiernos y que, como característica común en todos los países, muestran un gran aislamiento, desconexión y dificultad para posibles transferencias entre instituciones. (p. 5)

Además de lo anterior, existen otras dificultades derivadas de la necesidad de reconocer títulos provenientes de universidades extranjeras de manera ágil, en contraposición con el afán de garantizar que este reconocimiento sea producto de análisis exhaustivos, lo que causa conflictos en relación con la movilidad (Dávila, 2012). Se considera importante que exista un esfuerzo conjunto entre las universidades costarricenses para favorecer la movilidad de sus estudiantes al menos en el ámbito de docencia universitaria y educación.

\section{Gestión académica y liderazgo compartido}

Al referirse a la gestión, se hace necesario revisar los tres ámbitos de esta función directiva propuestos por Uribe (2010), a saber: institucional, curricular y de la convivencia:

i) La gestión institucional es el conjunto de acciones que aseguran la realización del proyecto educativo institucional. Dichas acciones del equipo directivo lideran la institución hacia metas explícitas, implican un proceso permanente de toma de decisiones generando un clima de trabajo colectivo y participativo....

ii) La gestión curricular es la capacidad de organizar y poner en marcha el proyecto pedagógico de la institución desde el punto de vista de lo que se requiere enseñar y de lo que es necesario que los estudiantes aprendan. Orienta y conduce las actividades del aula en el marco del proyecto educativo institucional, logrando trabajo en equipo de los docentes y generando las condiciones para que su trabajo se realice en un ambiente enriquecido y favorable a los aprendizajes....

iii) La gestión de la convivencia escolar: es el conjunto de acciones implicadas en la construcción de una convivencia escolar de calidad para sus integrantes, es decir, una gestión directiva que posibilite que los procesos educativos se desarrollen en un ambiente favorable, superando y previniendo conflictos que pueden afectar la calidad de vida de los miembros de la comunidad educativa. (pp. 311, 313) 
Cada ámbito de la gestión académica responde a la estructura funcional directiva y, para el caso de la maestría, ello recae en la persona coordinadora del posgrado, por lo que es ideal que se ejerza de manera conjunta con el liderazgo, entendido como el medio para fomentar el logro del éxito académico de la comunidad estudiantil y para la promoción del compromiso, la motivación y la participación activa del personal académico de la maestría, para favorecer el mejoramiento de la calidad educativa de esta misma. Por esto, se debe tomar en cuenta que el perfil directivo debe contemplar las competencias necesarias para la gestión de una educación de calidad en el contexto de las IES.

La gestión educativa como proceso implica la integración de una serie de aspectos, entre ellos los humanos, físicos, sociales, económicos, políticos y culturales, por lo que se hace necesario generar prácticas para la búsqueda de su efectividad y eficiencia en atención a lo definido en la misión y visión específicas y en función de los principios y valores institucionales. Tal y como lo afirma Pozner (2007):

Ser directivo y supervisor es trabajar en función de un horizonte de mejoramiento continuo, integrando teoría y conocimiento que proviene de la propia práctica, es unir lo que habitualmente se separa: ética con eficacia; y es primordialmente, mantener vivo el propósito moral de generar aprendizajes para todos. (pp. 71-72)

Lo anterior se relaciona con los planteamientos de la concepción del liderazgo compartido o bien liderazgo distribuido, que supone mucho más que una simple remodelación de tareas, significa, más bien, una transformación de la cultura institucional. Harris y Chapman (2002, citados por Murillo, 2006) expresan:

[Una] cultura, que [implica] el compromiso y la implicación de todos los miembros de la comunidad escolar en la marcha, el funcionamiento y la gestión de la escuela. De esta forma, el liderazgo distribuido aprovecha las habilidades de los otros en una causa común, de tal forma que el liderazgo se manifiesta a todos los niveles. (p. 10)

En esta visión de liderazgo, el rol del personal directivo pasa a ser el de agente de cambio que logra fusionar los recursos personales del equipo en función del logro del objetivo común del posgrado, promueve el desarrollo profesional y una práctica docente conjunta e integrada. Así lo expresa Murillo (2006):

Con el liderazgo distribuido se genera un incremento de la capacidad de la escuela para resolver sus problemas. Un centro se desarrolla cuando incrementa los aprendizajes de sus alumnos, reuniendo ... la labor en el aula [y conjunta]... del centro. La mejora del centro depende de la acción [conjunta] de los propios implicados. El directivo identifica, establece acuerdos y metas deseables, estimulando y desarrollando un clima de colaboración, apertura y confianza, lejos de la competitividad entre las distintas partes. (p. 10) 
doi: http://dx.doi.org/10.15359/ree.22-2.9

URL: http://www.una.ac.cr/educare

CORREO: educare@una.cr

Se reconoce que una gran mayoría del personal académico y administrativo, así como el estudiantado del programa de Maestría en Educación muestran un alto grado de compromiso con este programa de posgrado; ello, por la participación activa y vinculación con los diferentes procesos académicos desarrollados, por lo cual y según lo planteado anteriormente, se propone una gestión desvinculada del enfoque tradicional, en el cual la distribución del poder y de la autoridad se sitúa en un ente personal directivo. Esto permite el mayor aprovechamiento del recurso humano y que la gestión se convierta en un proceso donde el colectivo académico y estudiantil sea un protagonista que facilita el logro de objetivos comunes desde el desarrollo del compromiso y de responsabilidades conjuntas.

\section{Conclusiones y recomendaciones}

A partir del análisis de esta indagación, se puede manifestar que el programa de la Maestría en Educación de la Universidad Nacional ha llevado a cabo importantes esfuerzos por mejorar la calidad del personal docente de múltiples instituciones educativas y de diferentes niveles que han optado por titularse en este posgrado. Cada una de las conclusiones mencionadas a continuación responde a uno de los apartados de esta revisión bibliográfica.

Ejes curriculares. Se puede afirmar que el plan de estudios contempla los ejes curriculares recomendados para un programa de posgrado, lo cual evidencia una fortaleza de su diseño curricular.

Formación y prácticas docentes. En esta área, la maestría sigue siendo un posgrado pertinente y necesario en el contexto costarricense, ya que atiende la necesidad de contar con personal docente universitario con formación en pedagogía y andragogía como complemento de su disciplina.

En congruencia con la razón de ser de la Maestría en Educación del CIDE, es necesario que este programa vuelva su mirada al personal académico de la Universidad Nacional a través del fortalecimiento de procesos de vinculación que le permitan conocer y formar parte de este posgrado, pues constituye una herramienta fundamental en su quehacer académico.

Investigación y extensión. Se puede reconocer que la maestría en docencia universitaria fomenta los procesos investigativos tanto en su personal docente como en sus estudiantes, mas no sucede lo mismo con respecto a la extensión. En este sentido, es necesario que se desarrollen proyectos con los cuales este programa pueda tener un impacto positivo en las comunidades. Una de las posibilidades más destacables es que estos espacios se abran con base en los mismos procesos investigativos que tanto se promueven, y lleven la producción de conocimiento a la práctica. 
Acreditación. Dado que la acreditación de posgrados se traduce en transparencia y mejora continua de la calidad de los programas, llevar este proceso a la maestría en docencia universitaria, no solo ofrecida por la Universidad Nacional sino por la de Costa Rica y la ULACIT, resulta esencial. Esto incidiría también con las exigencias en las áreas de internacionalización y movilidad, ya que el ente regulador de estos procesos es de carácter internacional.

Internacionalización y movilidad. A pesar de la destacada labor que ha realizado la Maestría en Educación en relación con la formación de personal docente universitario, se considera necesario fortalecer los procesos de divulgación de este programa dentro de la misma universidad y fuera de ella, para abarcar las necesidades nacionales e internacionales, con el fin de que más académicos lo conozcan y aprovechen este valioso posgrado para mejorar su labor docente.

Se recomienda, también, un mayor aprovechamiento de la herramienta de la Universidad Nacional en su página web, para realizar esa divulgación del programa de Maestría en Educación con sus distintos énfasis que ofrece la División de Educología del CIDE, de manera que se incluyan la descripción de las carreras, planes de estudio, perfil del estudiantado graduado, vínculos de la Maestría con otros organismos, o cualquier otra información que se considere oportuna, con el fin de facilitar la movilidad estudiantil y docente.

Gestión académica y liderazgo compartido. Respecto a la gestión académica, se considera importante que la coordinación de la Maestría en Educación y de los posgrados en Docencia Universitaria logren un mejor aprovechamiento del recurso humano a través de las responsabilidades compartidas, donde las personas participantes del proceso educativo puedan aportar sus conocimientos y competencias para la mejora continua del programa y se comprometan con el desarrollo de liderazgos que promuevan el logro de objetivos de la educación superior.

\section{Referencias}

Agencia Centroamericana de Acreditación (ACAP). (2008). Manual de acreditación de la ACAP. Recuperado de http://www.utp.ac.pa/documentos/2013/pdf/ MANUALDEACREDITACIONACAP2013.pdf

Aguilar-Castillo, Y. y Riveros-Angarita, A. (2015). Gestión de la internacionalización en la Universidad de Costa Rica y su vínculo con Centroamérica y el Caribe. Revista Gestión de la Educación, 5(2), 99-135. doi: https://doi.org/10.15517/rge.v5i2.19968

Casas, M. (2005). Nueva universidad ante la sociedad del conocimiento. Revista de Universidad y Sociedad del Conocimiento, 2(2), 1-19. Recuperado de http://www.redalyc.org/articulo. oa?id=78020102 
doi: http://dx.doi.org/10.15359/ree.22-2.9

URL: http://www.una.ac.cr/educare

CORREO: educare@una.cr

Contreras, M. R., Alvarado, A., Maldonado, A. V. y Benítez, J. L. (2009). La evaluación del posgrado y su internacionalización. El caso de la universidad de occidente. Revista Educere et Educare, 4(8), 37-44. Recuperado de http://e-revista.unioeste.br/index.php/educereeteducare/ article/view/3548/2813

Dávila, M. (2012). Tendencias internacionales en posgrados. Núcleo de estudios e investigaciones en educación superior de MECOSUR, 1, 17-26. Recuperado de https://revistas.unc.edu.ar/ index.php/integracionyconocimiento/article/view/5630/6483

Declaración de Bolonia. Espacio Europeo de Enseñanza Superior. Declaración conjunta de los ministros europeos de enseñanza. (1999). Recuperado de http://www.eees.es/pdf/Bolonia ES.pdf

Ferro, J. (2014). Visión de la universidad ante el siglo XXI. Barranquilla: Editorial Universidad el Norte. Recuperado de https://www.uninorte.edu.co/documents/3055771/4f34a62b2651-433b-867a-62c8a1d772f5

González, J.y Santamaría, R. (2013). Calidad y acreditación en la educación superior: Integración e internacionalización de América Latina y el Caribe. Educación, 22(43), 131-147. Recuperado de http://www.revistas.pucp.edu.pe/index.php/educacion/article/viewFile/7500/7739

Lucarelli, E. (2004). Prácticas innovadoras en la formación del docente universitario. Educação, 27(54), 503-524. Recuperado de http://cmapspublic3.ihmc.us/rid=1216829834515 1063 $\underline{568960 \text { 5630/293.pdf }}$

Murillo, F. J. (2006). Una dirección escolar para el cambio: Del liderazgo transformacional al liderazgo distribuido. REICE. Revista Iberoamericana sobre Calidad, Eficacia y Cambio en Educación, 4(4e), 11-24. Recuperado de http://www.rinace.net/arts/vol4num4e/art2.pdf

Ortega, V. (2002). Tendencias de la educación universitaria en el siglo XXI. Arbor, 173(681), 67-81. doi: https://doi.org/10.3989/arbor.2002.i681.1109

Pozner, P. (2007). El papel de la supervisión en la mejora de la calidad de la educación. En M. A. Castañeda (Coord.), Seminario internacional Itinerante. Desarrollo de la gestión educativa en México: Situación actual y perspectivas (pp. 59-100). México: UPN. Recuperado de http://editorial.upnvirtual.edu.mx/index.php/publicaciones/9-publicaciones-upn/106seminario-internacional-itinerante

Programa Estado de la Nación. (2015). Capítulo 4: La evolución de la Educación Superior. En Quinto Informe Estado de la Educación. San José, Costa Rica: Autor. Recuperado de http:// www.estadonacion.or.cr/educacion2015/assets/cap-4-ee-2015.pdf 
Salomón, L. y Amador, J. (2013). Retos y desafíos de los posgrados en las universidades públicas del siglo XXI. Revista Ciencia y Tecnología, 12(1), 3-14. doi: https://doi.org/10.5377/rct. v0i12.1699

Tesouro, M., Corominas, E., Teixidó, J. y Puiggalí, J. (2014). La autoeficacia docente e investigadora del profesorado universitario: Relación con su estilo docente e influencia en sus concepciones sobre el nexo docencia-investigación. Revista de Investigación Educativa, 32(1), 169-186. doi: https://doi.org/10.6018/rie.32.1.172771

Universidad de Costa Rica, Sistema de Estudios de Posgrado. (s. f.). Maestría académica en educación con énfasis en docencia universitaria. Recuperado de http://www1.sep.ucr.ac.cr/ portal/135-areas/ciencias-sociales/educacion/docencia-universitaria.html

Universidad Latinoamericana de Ciencia y Tecnología. (s. f.). Maestría en currículum y docencia universitaria. Recuperado de http://www.ulacit.ac.cr/carreras/seccion/perfil. php? career $=5 \&$ grade $i d=5 \& i d=65$

Universidad Nacional. (s. f.). Maestría en Educación. Descripción. Recuperado de http://www.una. ac.cr/index.php/m-oferta-academica/educacion-maestria-en

Uribe, B. (2010). Profesionalizar la dirección escolar potenciando el liderazgo: Una clave ineludible en la mejora escolar. Desarrollo de perfiles de competencias directivas en el sistema educativo chileno. Revista Iberoamericana de Evaluación Educativa, 3(1), 303-322. Recuperado de https://revistas.uam.es/index.php/riee/article/view/4547/4981 